\title{
Trichophyton equinum
}

National Cancer Institute

\section{Source}

National Cancer Institute. Trichophyton equinum. NCI Thesaurus. Code C127752.

A species of zoophilic fungi in the phylum Ascomycota that causes ringworm in horses,

but rare infections in humans. 